\title{
The Applications of Cardinal Trigonometric Splines in Solving Nonlinear Integral Equations
}

\author{
Jin Xie, ${ }^{1}$ Xiaoyan Liu, ${ }^{2}$ and Lixiang $X u^{1}$ \\ ${ }^{1}$ Department of Mathematics and Physics, Hefei University, Hefei 230601, China \\ ${ }^{2}$ Department of Mathematics and Physics, University of La Verne, La Verne, CA 91750, USA \\ Correspondence should be addressed to Xiaoyan Liu; xliu@laverne.edu
}

Received 3 December 2013; Accepted 15 January 2014; Published 4 March 2014

Academic Editors: Y. M. Cheng and L. You

Copyright (c) 2014 Jin Xie et al. This is an open access article distributed under the Creative Commons Attribution License, which permits unrestricted use, distribution, and reproduction in any medium, provided the original work is properly cited.

\begin{abstract}
The cardinal trigonometric splines on small compact supports are employed to solve integral equations. The unknown function is expressed as a linear combination of cardinal trigonometric splines functions. Then a simple system of equations on the coefficients is deducted. When solving the Volterra integral equations, the system is triangular, so it is relatively straight forward to solve the nonlinear system of the coefficients and a good approximation of the original solution is obtained. The sufficient condition for the existence of the solution is discussed and the convergence rate is investigated.
\end{abstract}

\section{Introduction}

Trigonometric splines were introduced by Schoenberg in [1]. Univariate trigonometric splines are piecewise trigonometric polynomials of the form

$$
\sum_{k=0}^{n} a_{k} \cos \left(\alpha_{k} x\right)+b_{k} \sin \left(\alpha_{k} x\right) \quad\left(\text { where } b_{0}=0\right)
$$

(where $\alpha_{0}=0<\alpha_{1}<\alpha_{2}<\cdots<\alpha_{n}$ are real numbers) in each interval and they are nature extensions of polynomial splines. Needless to say, trigonometric splines have their own advantages. A number of papers have appeared to study the properties of the trigonometric splines and trigonometric Bsplines (cf. [2-4]) since then.

In my previous papers (cf. [5-7]), low degree orthonormal spline and cardinal spline functions with small compact supports were constructed. The method can be extended to construct higher degree orthonormal or cardinal splines. Unlike in the book (cf. [1]), by the cardinal splines we mean the specific splines satisfying cardinal interpolation conditions, which means that the cardinal function has the value one at one interpolation point and value zero at all other interpolation points. Cardinal splines are not only useful in interpolation problems, but they are also useful in deduction of numerical integration formulas [6] and in solving integral equations.

Integral equations appear in many fields, including dynamic systems, mathematical applications in economics, communication theory, optimization and optimal control systems, biology and population growth, continuum and quantum mechanics, kinetic theory of gases, electricity and magnetism, potential theory, and geophysics. Many differential equations with boundary value can be reformulated as integral equations. There are also some problems that can be expressed only in terms of integral equations.

In this paper we focus on the Volterra integral equations of the second kind:

$$
y(x)=g(x)+\lambda \int_{a}^{x} K(x, t) f(y(t)) d t, \quad x \in(a, b),
$$

where $\lambda$ is a complex number, the kernel $K(x, t), f(y)$, and $g(x)$ are known functions, and $y(x)$ is an unknown function to be determined.

This paper has six sections. In Section 2, a univariate trigonometric cardinal spline on a small compact support is constructed and properties are studied. In Section 3, the applications of trigonometric cardinal splines on solving the Volterra integral equations are explored. The unknown function is expressed as a linear combination of trigonometric 
cardinal spline functions. Then a simple system of nonlinear equations on the coefficients is deducted. It is relatively simple to solve the linear system since the system is triangular, and a good approximation of the original solution is obtained. The sufficient condition for the existence is discussed and the convergence rate is investigated. In Section 4, the applications of trigonometric cardinal splines on solving the systems of Volterra integral equations are explored. In Section 5, numerical examples are given on solving the nonlinear Volterra integral equations and a system of nonlinear Volterra integral equations. Section 6 contains the conclusion remarks.

\section{A Cardinal Trigonometric Spline with a Small Support}

To construct cardinal trigonometric splines, let

$$
\mu_{h}(x)= \begin{cases}1, & -\frac{h}{2}<x \leq \frac{h}{2} \\ 0, & \text { elsewhere. }\end{cases}
$$

This is the zero degree polynomial or trigonometric Bspline.

Let $T_{0, h}(x)=\mu_{h}(x)$. A continuous univariate cardinal trigonometric spline with a small support is

$$
T_{1, h}(x)=\frac{I_{1} T_{0}(x)}{I_{1} T_{0}(0)}=\frac{1}{2 \sin (h / 2)} \int_{-h / 2}^{h / 2} \mu_{h}(x+t) \cos t d t .
$$

Explicitly,

$$
T_{1, h}(x)=\left\{\begin{array}{r}
\frac{1}{2 \sin (h / 2)}\left(\sin \left(\frac{h}{2}\right)-\sin \left(x-\frac{h}{2}\right)\right), \\
\text { for } 0 \leq x \leq h, \\
\frac{1}{2 \sin (h / 2)}\left(\sin \left(\frac{h}{2}\right)+\sin \left(x+\frac{h}{2}\right)\right), \\
\text { for }-h \leq x \leq 0, \\
0, \quad \text { for } x>h \text { or } x<-h .
\end{array}\right.
$$

The graph of $T_{1, h}(x)$ is Figure 1.

Proposition 1. If $y(x) \in C^{1}[a, b], y^{\prime \prime}(x)$ exists and is bounded on the finite interval $[a, b]$ (where $a<b)$, for any $x \in[a, b]$ and any integer $n$, such that $h:=(b-a) / n<1$; let

$$
T L y(x)=\sum_{j=0}^{n} y(a+j h) T_{1, h}(x-a-j h)
$$

then $|y(x)-T L y((x))| \leq 6 h^{2} \operatorname{Max}_{x \in[a, b]}\left|y^{\prime \prime}(x)\right|$.

If $y(x) \in C^{1}(-\infty, \infty), y^{\prime \prime}(x)$ exists on $(-\infty, \infty)$ and both $y^{\prime}(x)$ and $y^{\prime \prime}(x)$ are bounded, for any $x \in(-\infty, \infty)$ and any chosen $h<1$, let

$$
T L(y(x))=\sum_{j=-\infty}^{\infty} y(j h) T_{1, h}(x-j h)
$$

then $|y(x)-T L y((x))| \leq 6 h^{2} \operatorname{Max}_{x \in(-\infty, \infty)}\left|y^{\prime \prime}(x)\right|$.



Figure 1: The graph of $T_{1, h}(x)$.

\section{Numerical Method Solving Integral Equations}

To solve the Volterra integral equations (2) in an interval $(a, b)$, we let $h=(b-a) / n, x_{i}=a+i h, i=0,1, \ldots, n$. Furthermore, let

$$
\begin{gathered}
y(x)=\sum_{k=0}^{n} c_{k} T_{1, h}\left(x-x_{k}\right), \\
f(y(x))=\sum_{k=0}^{n} f\left(c_{k}\right) T_{1, h}\left(x-x_{k}\right), \\
K(x, t)=\sum_{i=0}^{n} \sum_{j=0}^{n} K\left(x_{i}, x_{j}\right) T_{1, h}\left(x-x_{i}\right) T_{1, h}\left(t-x_{j}\right), \\
g(x)=\sum_{k=0}^{n} g\left(x_{k}\right) T_{1, h}\left(x-x_{k}\right) ;
\end{gathered}
$$

plugging in (2), we get

$$
\begin{aligned}
& \sum_{k=0}^{n} c_{k} T_{1, h}\left(x-x_{k}\right) \\
&-\lambda \sum_{i=0}^{n} \sum_{j=0}^{n} \sum_{k=0}^{n} T_{1, h}\left(x-x_{i}\right) \int_{a}^{x} K\left(x_{i}, x_{j}\right) T_{1, h}\left(t-x_{j}\right) \\
& f\left(c_{k}\right) T_{1, h}\left(t-x_{k}\right) d t
\end{aligned}
$$

$$
=\sum_{k=0}^{n} g\left(x_{k}\right) T_{1, h}\left(x-x_{k}\right) \text {. }
$$

Letting $x=x_{k_{0}}$, we arrive for $k_{0}=0,1,2,3,4, \ldots, n$, (where $\alpha=\int_{-\infty}^{\infty} T_{1,1}(x) T_{1,1}(x) d x, \beta=\int_{-\infty}^{\infty} T_{1,1}(x) T_{1,1}(x-$ 1) $d x$, ) at

$$
\begin{aligned}
g\left(x_{k_{0}}\right)= & c_{k_{0}}-\lambda\left(\frac{\alpha}{2} f\left(c_{0}\right)+\beta f\left(c_{1}\right)\right) h K\left(x_{0}, x_{k_{0}}\right) \\
& -\sum_{i=1}^{k_{0}-1} \lambda\left(\beta f\left(c_{i-1}\right)+\alpha f\left(c_{i}\right)+\beta f\left(c_{i+1}\right)\right) h K\left(x_{i}, x_{k_{0}}\right) \\
& -\lambda\left(\beta f\left(c_{k_{0}-1}\right)+\frac{\alpha}{2} f\left(c_{k_{0}}\right)\right) h K\left(x_{k_{0}}, x_{k_{0}}\right)
\end{aligned}
$$


which is a triangular system of $n+1$ nonlinear equations on unknowns $c_{0}, c_{1}, \ldots, c_{n}$. Notice that the coefficient matrix for the system is a triangular matrix, which means that we solve $c_{i}=f\left(c_{i}\right)+W_{i}$, where $W_{i}$ is a number not depending on $c_{i}$, for $i=0,1, \ldots, n$. For the convergence rate of solution of the Volterra integral equations (2), we have the following Proposition 2.

Proposition 2. Given that $y(x), g(x) \in C[a, b], y^{\prime \prime}(x)$, and $g^{\prime \prime}(x)$ exist and are bounded in $[a, b], K(x, y) \in C[a, b] \times[a, b]$, $\left(\partial^{2} / \partial x^{s} \partial y^{t}\right) K(x, y)(s+t=2)$ exist and are bounded in $[a, b] \times$ $[a, b]$. Furthermore, $K(x, y)$ satisfies the condition

$$
\left|\lambda \int_{a}^{b} K(x, y)(y(x)-u(x)) d x\right|<L M \max _{x \in[a, b]}|y(x)-u(x)|,
$$

where $|L M|<1$. Let $n$ be an integer, $h=(b-a) / n$, let $x_{i}=$ $a+i h, y_{i}=y\left(x_{i}\right), i=0,1,2, \ldots, n$, and $c_{0}, c_{1}, \ldots, c_{n}$ satisfies the linear system $(S 1)$

$$
y^{*}(x)=\sum_{k=0}^{n} c_{i} T_{1, h}(x-k h)
$$

then

$$
\left\|y^{*}(x)-y(x)\right\|_{[a, b]}=O\left(h^{2}\right),
$$

where $y(x)$ is the exact solution of (2).

Proof. Let

$$
\begin{gathered}
y(x)=\sum_{k=0}^{n} c_{k} T_{1, h}\left(x-x_{k}\right), \\
f(y(t))=\sum_{k=0}^{n} f\left(c_{k}\right) T_{1, h}\left(x-x_{k}\right), \\
K(x, t)=\sum_{i=0}^{n} \sum_{j=0}^{n} K\left(x_{i}, x_{j}\right) T_{1, h}\left(x-x_{i}\right) T_{1, h}\left(t-x_{j}\right), \\
g(x)=\sum_{k=0}^{n} g\left(x_{k}\right) T_{1, h}\left(x-x_{k}\right),
\end{gathered}
$$

where the coefficients are the solutions of above system $(S 1)$. Then

$$
\begin{aligned}
\| f(x) & -f^{*}(x) \|_{[a, b]} \\
=\| & \lambda \int_{a}^{b} K(x, t) f(t) d t+g(x)-\lambda \int_{a}^{b} K^{*}(x, t) f^{*}(t) d t \\
& \quad-g^{*}(x) \|_{[a, b]}
\end{aligned}
$$

$$
\begin{aligned}
=\| & \lambda \int_{a}^{b} K(x, t) f(t) d t-\lambda \int_{a}^{b} K^{*}(x, t) f(t) d t \\
& +\lambda \int_{a}^{b} K^{*}(x, t) f(t) d t-\lambda \int_{a}^{b} K^{*}(x, t) f^{*}(t) d t \\
& +g(x)-g^{*}(x) \|_{[a, b]} \\
= & \| \lambda \int_{a}^{b}\left(K(x, t)-K^{*}(x, t)\right) f(t) d t \\
& +\lambda \int_{a}^{b} K^{*}(x, t)\left(f(t)-f^{*}(t)\right) d t+\left(g(x)-g^{*}(x)\right) \|_{[a, b]} \\
\leq & 48|\lambda| \max _{s+t=2}\left\{\left\|\frac{\partial^{2}}{\partial x^{s} \partial y^{t}} K(x, y)\right\|_{[a, b] \times[a, b]}\right\} \\
& \times\|f(x)\|_{[a, b]}(b-a) h^{2} \\
& +L M\left\|f(x)-f^{*}(x)\right\|_{[a, b]}+\left\|g(x)-g^{*}(x)\right\|_{[a, b]} .
\end{aligned}
$$

Plug in

$$
\|f(x)\|_{[a, b]} \leq \frac{1}{1-L M}\|g(x)\|_{[a, b]} .
$$

Therefore

$$
\begin{aligned}
\left\|f(x)-f^{*}(x)\right\|_{[a, b]} & \\
\leq \frac{1}{1-L M}( & \frac{48|\lambda|(b-a)}{1-L M} \\
& \quad \times \max _{s+t=2}\left\{\left\|\frac{\partial^{2}}{\partial x^{s} \partial y^{t}} K(x, y)\right\|_{[a, b] \times[a, b]}\right\} \\
& \left.\times\|g(x)\|_{[a, b]}+7\left\|g^{\prime \prime}(x)\right\|_{[a, b]}\right) h^{2} .
\end{aligned}
$$

\section{Numerical Method Solving Systems of Integral Equations}

The system of Volterra integral equations is critical to many physical, biological, and engineering models. For instance, for some heat transfer problems in physics, the heat equations are usually replaced by a system of Volterra integral equations [8]. Many well-known models for neural networks in biomathematics, nuclear reactor dynamics problems, and thermoelasticity problems are also based on a system of Volterra integral equations $([9,10])$. Our method could be extended to solve the system of Volterra integral equations. Given

$$
\begin{array}{r}
y_{s}(x)=g_{s}(x)+\sum_{p=0}^{m} \lambda_{p} \int_{a}^{x} K_{p, s}(x, t) f_{p, s}\left(y_{p}(t)\right) d t, \\
x \in(a, b), \quad s=1,2, \ldots, m,
\end{array}
$$


in an interval $(a, b)$, we let $h=(b-a) / n, x_{i}=a+i h, i=$ $0,1, \ldots, n$. Furthermore, let $(s, p=1,2, \ldots, m)$

$$
\begin{gathered}
y_{p}(x)=\sum_{k=0}^{n} c_{k, p} T_{1, h}\left(x-x_{k}\right), \\
f_{p, s}\left(y_{p}(x)\right)=\sum_{k=0}^{n} f_{p, s}\left(c_{k, p}\right) T_{1, h}\left(x-x_{k}\right), \\
K_{p, s}(x, t)=\sum_{i=0}^{n} \sum_{j=0}^{n} K_{p, s}\left(x_{i}, x_{j}\right) T_{1, h}\left(x-x_{i}\right) T_{1, h}\left(t-x_{j}\right), \\
g_{s}(x)=\sum_{k=0}^{n} g_{s}\left(x_{k}\right) T_{1, h}\left(x-x_{k}\right) ;
\end{gathered}
$$

plugging in (17), we get

$$
\begin{aligned}
& \sum_{k=0}^{n} c_{k, s} T_{1, h}\left(x-x_{k}\right)-\sum_{p=0}^{m} \lambda_{p} \sum_{i=0}^{n} \sum_{j=0}^{n} \sum_{k=0}^{n} T_{1, h}\left(x-x_{i}\right) \\
& \quad \int_{a}^{x} K_{p, s}\left(x_{i}, x_{j}\right) T_{1, h}\left(t-x_{j}\right) f_{p, s}\left(c_{k, p}\right) T_{1, h}\left(t-x_{k}\right) d t \\
& =\sum_{k=0}^{n} g_{s}\left(x_{k}\right) T_{1, h}\left(x-x_{k}\right) .
\end{aligned}
$$

Let $x=x_{k_{0}}$, we arrive for $k_{0}=0,1,2,3,4, \ldots, n$, (where $\left.\alpha=\int_{-\infty}^{\infty} T_{1,1}(x) T_{1,1}(x) d x, \beta=\int_{-\infty}^{\infty} T_{1,1}(x) T_{1,1}(x-1) d x,\right)$ at

$g_{s}\left(x_{k_{0}}\right)$

$$
\begin{aligned}
& =c_{k_{0}, s}-\sum_{p=0}^{m} \lambda_{p}\left(\frac{\alpha}{2} f_{p, s}\left(c_{0, s}\right)+\beta f_{p, s}\left(c_{1, s}\right)\right) \\
& \quad \times h K_{p, s}\left(x_{0}, x_{k_{0}}\right) \\
& -\sum_{p=0}^{m} \lambda_{p} \sum_{i=1}^{k_{0}-1}\left(\beta f_{p, s}\left(c_{i-1, s}\right)+\alpha f_{p, s}\left(c_{i, s}\right)+\beta f_{p, s}\left(c_{i+1, s}\right)\right) \\
& \quad \times h K_{p, s}\left(x_{i}, x_{k_{0}}\right) \\
& -\sum_{p=0}^{m} \lambda_{p}\left(\beta f_{p, s}\left(c_{k_{0}-1, s}\right)+\frac{\alpha}{2} f_{p, s}\left(c_{k_{0}, s}\right)\right) h K_{p, s}\left(x_{k_{0}}, x_{k_{0}}\right),
\end{aligned}
$$

which is a triangular system of $(n+1) m$ nonlinear equations on unknowns $\left\{c_{0, s}, c_{1, s}, \ldots, c_{n, s}\right\}$. Notice that the coefficient matrix for the system is a triangular matrix, which means that we solve $c_{i, s}=f_{p, s}\left(c_{i, s}\right)+W_{i, s}$, where $W_{i}$ is a number, for $i=$ $0,1, \ldots, n$. For the convergence rate of solution of the Volterra integral equations (2), we have the following Proposition 3.

Proposition 3. Given that $y_{p}(x), g_{p}(x) \in C[a, b], y_{p}^{\prime \prime}(x)$, and $g_{p}^{\prime \prime}(x)$ exists and is bounded in $[a, b], K_{p, s}(x, y) \in C[a, b] \times$ $[a, b],\left(\partial^{2} / \partial x^{s} \partial y^{t}\right) K_{p, s}(x, y)(s+t=2)$ exist and are bounded in $[a, b] \times[a, b]$. Furthermore, $K_{p, s}(x, y)$ satisfies the condition

$$
\begin{gathered}
\left|\sum_{p=0}^{m} \lambda_{p} \int_{a}^{b} K_{p, s}(x, y)\left(y_{p}(x)-u_{p}(x)\right) d x\right| \\
<L M \max _{x \in[a, b]} \sum_{p=0}^{m}\left|\lambda_{p}\right|\left|y_{p}(x)-u_{p}(x)\right|,
\end{gathered}
$$

where $|L M|<1$. Let $n$ be an integer, $h=(b-a) / n$, let $x_{i}=$ $a+i h, y_{i}=y\left(x_{i}\right), i=0,1,2, \ldots, n, c_{0, p}, c_{1, p}, \ldots, c_{n, p}$ satisfies the linear system (S1)

$$
y_{p}^{*}(x)=\sum_{k=0}^{n} c_{i, p} T_{1, h}(x-k h) ;
$$

then

$$
\left\|y_{p}^{*}(x)-y_{p}(x)\right\|_{[a, b]}=O\left(h^{2}\right)
$$

where $\left\{y_{p}(x)\right\}$ is the exact solution of (17).

\section{Numerical Examples}

Example 1. Given that $y(x)=g(x)+\int_{0}^{x} K(x, t) f(y(t)) d t$, where $k(x, t)=\tan (x-t) /(\sqrt{t}+0.1)^{2}, f(y)=y^{2}, g(x)=$ $\sqrt{x}+0.1+\ln |\cos x|$.

Let $(a, b)=(0,1), n=10, h=0.1, x_{i}=$ ih. $y(x)=$ $\sum_{k=0}^{10} c_{k} T_{1, h}\left(x-x_{k}\right), y^{2}(t)=\sum_{k=0}^{10} c_{k}^{2} T_{1, h}\left(x-x_{k}\right), K(x, t)=$ $\tan (x-t) /(\sqrt{t}+0.1)^{2}=\sum_{i=0}^{10} \sum_{j=0}^{10}\left(\tan \left(x_{i}-x_{j}\right) /\left(\sqrt{x_{j}}+\right.\right.$ $\left.0.1)^{2}\right) T_{1, h}\left(x-x_{i}\right) T_{1, h}\left(t-x_{j}\right), g(x)=\sum_{k=0}^{10} g\left(x_{k}\right) T_{1, h}\left(x-x_{k}\right)$. Plugging into the integral equation, we arrive at

$$
\begin{gathered}
c_{0}=g\left(x_{0}\right), \\
g\left(x_{k}\right)=c_{k}-\left(\frac{\alpha}{2} c_{0}^{2}+\beta c_{1}^{2}\right) h K\left(x_{0}, x_{k}\right) \\
-\sum_{i=1}^{k-1}\left(\beta c_{i-1}^{2}+\alpha c_{i}^{2}+\beta c_{i+1}^{2}\right) h K\left(x_{i}, x_{k}\right) \\
-\left(\beta c_{k-1}^{2}+\frac{\alpha}{2} c_{k}^{2}\right) h K\left(x_{k}, x_{k}\right), \quad(k=1,2, \ldots, 10) .
\end{gathered}
$$

The solution is $\left[c_{0}, c_{1}, c_{2}, c_{3}, c_{4}, c_{5}, c_{6}, c_{7}, c_{8}, c_{9}, c_{10}\right]$ $=[0.4162361433,0.5472481952,0.6478042689,0.7326106099$, $0.8073700605,0.8750164888,0.9373063268,0.9954057887$, $1.050163033,1.102269297]$.

Compared with the exact solution [0.4162277660, $0.5472135955,0.6477225575,0.7324555320,0.8071067811$, $0.8745966692,0.9366600265,0.9944271910,1.048683298$, 1.1]. The error $E<0.003=3 h^{3}$.

Example 2. $u(x)=\tan x-(1 / 4) \sin (2 x)-(1 / 2) x+\int_{-1 / 2}^{x} 1 /(1+$ $\left.u^{2}(t)\right) d t,-1 / 2 \leq x \leq 1 / 2$, with the exact solution $u(x)=$ $\tan x$. 
We choose $n=10, h=0.1$, and $x_{k}=-(1 / 2)+k h$, and let $u^{*}(x)=\sum_{k=0}^{10} c_{k} T_{1, h}\left(x-x_{k}\right)$ plug into $(S 1)$. The system has the form

$$
\begin{aligned}
c_{0}=g\left(x_{0}\right) \\
g\left(x_{k}\right)=c_{k}-\left(\frac{\alpha}{2} \frac{1}{\left(c_{0}^{2}+1\right)}+\frac{\beta}{\left(c_{1}^{2}+1\right)}\right) h \\
-\sum_{i=1}^{k-1}\left(\frac{\beta}{\left(c_{i-1}^{2}+1\right)}+\frac{\alpha}{\left(c_{i}^{2}+1\right)}+\frac{\beta}{\left(c_{i+1}^{2}+1\right)}\right) h \\
-\left(\frac{\beta}{\left(c_{k-1}^{2}+1\right)}+\frac{\alpha}{2} \frac{1}{\left(c_{k}^{2}+1\right)}\right) h \\
(k=1,2, \ldots, 10) .
\end{aligned}
$$

The solution is $\left[c_{0}, c_{1}, c_{2}, c_{3}, c_{4}, c_{5}, c_{6}, c_{7}, c_{8}, c_{9}, c_{10}\right]$ $=[-0.5463024898,-0.4228999647,-0.3095798686$, $-0.2031136045, \quad-0.1009105373, \quad-0.0007472398948$, $0.09943058913,0.201675057,0.308203618,0.4215987172$, 0.5450804126]. Comparing with the exact solutions $\left[u\left(x_{0}\right), u\left(x_{1}\right), u\left(x_{2}\right), u\left(x_{3}\right), u\left(x_{4}\right), u\left(x_{5}\right), u\left(x_{6}\right), u\left(x_{7}\right)\right.$, $\left.u\left(x_{8}\right), u\left(x_{9}\right), u\left(x_{10}\right)\right]=[-0.5463024898,-0.4227932187$, $-0.3093362496, \quad-0.2027100355, \quad-0.1003346720, \quad 0$, $0.1003346720,0.2027100355,0.3093362496,0.4227932187$, $0.5463024898]$, the error $E<0.002$.

Notice that our accuracy is much better than the one in the paper [11] although they choose $h=0.01$ in the paper.

Example 3. The equation of percolation in [12]

$$
y(x)=\int_{0}^{x} e^{A(x-t)}(1+(x-t) \ln A)(y(t))^{1 / p} d t,
$$

where $A>1$ physical parameter, constant $p>1$; according to paper [13], we can obtain a unique nonnegative nontrivial solution. Let $(a, b)=(0,1), n=10, h=0.1, x_{i}=i h$. $y(x)=\sum_{k=0}^{10} c_{k} T_{1, h}\left(x-x_{k}\right), K(x, t)=e^{A(x-t)}(1+(x-t) \ln A)=$ $\sum_{i=0}^{10} \sum_{j=0}^{10} e^{A\left(x_{i}-x_{j}\right)}\left(1+\left(x_{i}-x_{j}\right) \ln A\right) T_{1, h}\left(x-x_{i}\right) T_{1, h}\left(t-x_{j}\right)$, $g(x)=0$. Let $A=2, p=2$; plugging into the integral equation, we arrive at

$$
\begin{gathered}
c_{0}=0 \\
0=c_{k}-\left(\frac{\alpha}{2} c_{0}^{1 / p}+\beta c_{1}^{1 / p}\right) h K\left(x_{0}, x_{k}\right) \\
-\sum_{i=1}^{k-1}\left(\beta c_{i-1}^{1 / p}+\alpha c_{i}^{1 / p}+\beta c_{i+1}^{1 / p}\right) h K\left(x_{i}, x_{k}\right) \\
-\left(\beta c_{k-1}^{1 / p}+\frac{\alpha}{2} c_{k}^{1 / p}\right) h K\left(x_{k}, x_{k}\right) \\
(k=1,2, \ldots, 10) .
\end{gathered}
$$

The result we got is $\left[c_{0}, c_{1}, c_{2}, c_{3}, c_{4}, c_{5}, c_{6}, c_{7}, c_{8}, c_{9}, c_{10}\right]=$ $[0,0.003026,0.013703,0.035223,0.071853,0.129210,0.214645$, $0.337732,0.510886,0.750148,1.076178]$.
We do not have the exact solution. Nevertheless, compare with $y\left(x_{k}\right)=\int_{0}^{x_{k}} e^{A\left(x_{k}-t\right)}\left(1+\left(x_{k}-t\right) \ln A\right)(y(t))^{1 / p} d t,\left[y\left(x_{0}\right)\right.$, $y\left(x_{1}\right), y\left(x_{2}\right), y\left(x_{3}\right), y\left(x_{4}\right), y\left(x_{5}\right), y\left(x_{6}\right), y\left(x_{7}\right), y\left(x_{8}\right), y\left(x_{9}\right)$, $\left.y\left(x_{10}\right)\right]=[0,0.003011,0.013632,0.035039,0.071478,0.128539$, $0.213538,0.336002,0.508289,0.746365,1.070795]$.

For the same integral equation, let $A=2, p=$ 3 ; we arrive at $\left[c_{0}, c_{1}, c_{2}, c_{3}, c_{4}, c_{5}, c_{6}, c_{7}, c_{8}, c_{9}, c_{10}\right]=$ [0.012903, 0.051423,0.116203,0.213289, 0.351521, 0.542618, $0.801744,1.148333,1.607165,2.209747]$.

Compare with $y\left(x_{k}\right)=\int_{0}^{x_{k}} e^{A\left(x_{k}-t\right)}\left(1+\left(x_{k}-\right.\right.$ $t) \ln A)(y(t))^{1 / p} d t, \quad\left[y\left(x_{0}\right), \quad y\left(x_{1}\right), \quad y\left(x_{2}\right), \quad y\left(x_{3}\right), \quad y\left(x_{4}\right)\right.$, $\left.y\left(x_{5}\right), y\left(x_{6}\right), y\left(x_{7}\right), y\left(x_{8}\right), y\left(x_{9}\right), y\left(x_{10}\right)\right]=[0.012840$, $0.051156,0.115595,0.212177,0.349700,0.539832,0.797666$, $1.142548,1.599148,2.198834]$.

Example 4. Consider the equation $y(x)-(1 /$ 6) $\int_{0}^{x} \sin (y(t)) \cos (x-t) d t=(1 / 6) \cos x+\arcsin x-(1 / 6)=$ $g(x)$.

The exact solution is $y=\arcsin x$.

Applying the method on the interval $[0,1]$, let $h=1 / 10$, $n=10, x_{i}=i h, K(x, t)=\cos (x-t)=\sum_{i=-1}^{11} T_{1, h}(x-$ $\left.x_{i}\right) \sum_{j=-1}^{11} \cos \left(x_{i}-t_{j}\right) T_{1, h}\left(t-x_{j}\right), g(x)=(1 / 6) \cos x+\arcsin x-$ $(1 / 6)=\sum_{i=-1}^{11} g\left(x_{i}\right) T_{1, h}\left(x-x_{i}\right)$, and $y(x)=\sum_{k=-1}^{n+1} c_{k} T_{1, h}(x-$ $\left.x_{k}\right)$; plugging into the integral equation (2), we arrive at

$$
\begin{gathered}
c_{0}=g\left(x_{0}\right), \\
g\left(x_{k}\right)=c_{k}-\lambda\left(\frac{\alpha}{2} \sin \left(c_{0}\right)+\beta \sin \left(c_{1}\right)\right) h K\left(x_{0}, x_{k}\right) \\
-\sum_{i=1}^{k-1} \lambda\left(\beta \sin \left(c_{i-1}\right)+\alpha \sin \left(c_{i}\right)\right. \\
\left.+\beta \sin \left(c_{i+1}\right)\right) h K\left(x_{i}, x_{k}\right) \\
-\lambda\left(\beta \sin \left(c_{k-1}\right)+\frac{\alpha}{2} \sin \left(c_{k}\right)\right) h K\left(x_{k}, x_{k}\right), \\
\quad(k=1,2, \ldots, 10) .
\end{gathered}
$$

The solution is $\left[c_{0}, c_{1}, c_{2}, c_{3}, c_{4}, c_{5}, c_{6}, c_{7}, c_{8}, c_{9}, c_{10}\right]=$ $[0.1001681505,0.2013552395,0.3046865884,0.4115060574$, $0.5235819595,0.6434770162,0.775364957,0.9272531678$, $1.119717038,1.570732818]$.

Compared with the exact solution: $\left[y\left(x_{0}\right), y\left(x_{1}\right), y\left(x_{2}\right)\right.$, $\left.y\left(x_{3}\right), y\left(x_{4}\right), y\left(x_{5}\right), y\left(x_{6}\right), y\left(x_{7}\right), y\left(x_{8}\right), y\left(x_{9}\right), y\left(x_{10}\right)\right]$ $=[0.1001674211,0.2013579207,0.3046926540,0.4115168460$, $0.5235987755,0.6435011087,0.7753974965,0.9272952180$, $1.119769515,1.570796326]$. The error $E<0.00007$.

Example 5. Consider the equation $y(x)-(1 / 8) \int_{1}^{x}(1 /$ $\left.\sqrt{x^{2}+t^{2}}\right) e^{y(t)} d t=g(x)=\ln x-(1 / 8) x \sqrt{2}+(1 / 8) \sqrt{x^{2}+1}$; the exact solution is $y(x)=\ln x$.

Applying the method on the interval $[1,2]$, let $h=$ $1 / 10, n=10, x_{i}=1+i h, K(x, t)=1 / \sqrt{x^{2}+t^{2}}=$ $\sum_{i=-1}^{11} T_{1, h}\left(x-x_{i}\right) \sum_{j=-1}^{11} K\left(x_{i}, x_{j}\right) T_{1, h}\left(t-x_{j}\right), g(x)=\ln x-$ $(1 / 8) x \sqrt{2}+(1 / 8) \sqrt{x^{2}+1}=\sum_{i=-1}^{11} g\left(x_{i}\right) T_{1, h}\left(x-x_{i}\right)$, and 
$y(x)=\sum_{k=-1}^{n+1} c_{k} T_{1, h}\left(x-x_{k}\right)$; plugging into the integral equation, we arrive at

$$
\begin{gathered}
c_{0}=g\left(x_{0}\right), \\
g\left(x_{k}\right)=c_{k}-\lambda\left(\frac{\alpha}{2} e^{c_{0}}+\beta e^{c_{1}}\right) h K\left(x_{0}, x_{k}\right) \\
-\sum_{i=1}^{k-1} \lambda\left(\beta e^{c_{i-1}}+\alpha e^{c_{i}}+\beta e^{c_{i+1}}\right) h K\left(x_{i}, x_{k}\right) \\
-\lambda\left(\beta e^{c_{k-1}}+\frac{\alpha}{2} e^{c_{k}}\right) h K\left(x_{k}, x_{k}\right), \\
(k=1,2, \ldots, 10) .
\end{gathered}
$$

The solution is $\left[c_{0}, c_{1}, c_{2}, c_{3}, c_{4}, c_{5}, c_{6}, c_{7}, c_{8}, c_{9}\right.$, $\left.c_{10}\right]=[0.09531139,0.18232333,0.2623661939,0.3364740924$, $0.4054667723,0.4700050464,0.5306294034,0.5877875548$, $0.6418545277,0.6931475935]$.

Compared with the exact solution $\left[y\left(x_{0}\right), y\left(x_{1}\right), y\left(x_{2}\right)\right.$, $\left.y\left(x_{3}\right), y\left(x_{4}\right), y\left(x_{5}\right), y\left(x_{6}\right), y\left(x_{7}\right), y\left(x_{8}\right), y\left(x_{9}\right), y\left(x_{10}\right)\right]=$ [0.09531017980, 0.1823215567, 0.2623642644, 0.3364722366, $0.4054651081,0.4700036292,0.5306282510,0.5877866649$, $0.6418538861,0.6931471805]$. The error $E<0.000001$.

Example 6. Consider a system of Volterra integral equations:

$$
\begin{aligned}
& u(x)=\sin x-x+\int_{0}^{x}\left(u^{2}(t)+v^{2}(t)\right) d t \\
& v(x)=\cos x-\frac{1}{2} \sin ^{2} x+\int_{0}^{x}(u(t) v(t)) d t
\end{aligned}
$$

The exact solutions are $u(x)=\sin x, v(x)=\cos x$.

In an interval $(0,1)$, we let $h=1 / 10, x_{i}=i h, i=$ $0,1, \ldots, 10$. Furthermore, let

$$
\begin{gathered}
u(x)=\sum_{k=0}^{n} c_{k, 1} T_{1, h}\left(x-x_{k}\right), \\
v(x)=\sum_{k=0}^{n} c_{k, 2} T_{1, h}\left(x-x_{k}\right), \\
(u(x))^{2}=\sum_{k=0}^{n}\left(c_{k, 1}\right)^{2} T_{1, h}\left(x-x_{k}\right), \\
(v(x))^{2}=\sum_{k=0}^{n}\left(c_{k, 2}\right)^{2} T_{1, h}\left(x-x_{k}\right), \\
(u(x) v(x))=\sum_{k=0}^{n}\left(c_{k, 1} c_{k, 2}\right) T_{1, h}\left(x-x_{k}\right), \\
K(x, t)=1=\sum_{i=0}^{n} \sum_{j=0}^{n} T_{1, h}\left(x-x_{i}\right) T_{1, h}\left(t-x_{j}\right),
\end{gathered}
$$

$$
\begin{gathered}
g(x)=\sin x-x=\sum_{k=0}^{n} g\left(x_{k}\right) T_{1, h}\left(x-x_{k}\right), \\
h(x)=\cos x-\frac{1}{2} \sin ^{2} x=\sum_{k=0}^{n} h\left(x_{k}\right) T_{1, h}\left(x-x_{k}\right) ;
\end{gathered}
$$

plugging into the system, we get

$$
\begin{aligned}
& c_{0,1}=0 \text {, } \\
& g\left(x_{k}\right)=c_{k, 1}-\left(\frac{\alpha}{2}\left(c_{0,1}^{2}+c_{0,2}^{2}\right)+\beta\left(c_{1,1}^{2}+c_{1,2}^{2}\right)\right) h \\
& -\sum_{i=1}^{k-1}\left(\beta\left(c_{i-1,1}^{2}+c_{i-1,2}^{2}\right)+\alpha\left(c_{i, 1}^{2}+c_{i, 2}^{2}\right)\right. \\
& \left.+\beta\left(c_{i+1,1}^{2}+c_{i+1,2}^{2}\right)\right) h \\
& -\left(\beta\left(c_{k-1,1}^{2}+c_{k-1,2}^{2}\right)+\frac{\alpha}{2}\left(c_{k, 1}^{2}+c_{k, 2}^{2}\right)\right) h, \\
& (k=1,2, \ldots, 10) \text {, } \\
& c_{0,2}=1 \text {, } \\
& h\left(x_{k}\right)=c_{k, 2}-\left(\frac{\alpha}{2}\left(c_{0,1} c_{0,2}\right)+\beta\left(c_{1,1} c_{1,2}\right)\right) h \\
& -\sum_{i=1}^{k-1}\left(\beta\left(c_{i-1,1} c_{i-1,2}\right)+\alpha\left(c_{i, 1} c_{i, 2}\right)+\beta\left(c_{i+1,1} c_{i+1,2}\right)\right) h \\
& -\left(\beta\left(c_{k-1,1} c_{k-1,2}\right)+\frac{\alpha}{2}\left(c_{k, 1} c_{k, 2}\right)\right) h, \\
& (k=1,2, \ldots, 10) \text {, }
\end{aligned}
$$

which is a nearly triangular system of $(10+1) 2$ nonlinear equations on unknowns $\left\{\left[c_{0,1}, c_{1,1}, \ldots, c_{n, 1}\right],\left[c_{0,2}, c_{1,2}\right.\right.$, $\left.\left.\ldots, c_{n, 2}\right]\right\}$; we need to solve two nonlinear equations: $c_{i, 1}=$ $f_{1}\left(c_{i, 1}, c_{i, 2}\right)+W_{i, 1}, c_{i, 2}=f_{2}\left(c_{i, 1}, c_{i, 2}\right)+W_{i, 2}$, where $W_{i, 1}, W_{i, 1}$ are numbers, each time for $i=0,1, \ldots, n$.

Solutions are $\left\{\left[c_{0,1}, c_{1,1}, \ldots, c_{n, 1}\right],\left[c_{0,2}, c_{1,2}, \ldots, c_{n, 2}\right]\right\}=$ $\{[0.09983172966,0.1986591558,0.2954877407,0.3893423816$, $0.4792769003,0.5643832656,0.6438004752,0.7167230387$, 0.782409006, 0.8405334929], [0.9949873745, 0.9799992481, $0.9551843698,0.9207890215,0.8771545950,0.8247142533$, $0.7639887345,0.6955813657,0.6201723633,0.5443657395]\}$. Compared with the exact solution: $\{[0,0.09983341664$, $0.1986693307,0.2955202066,0.3894183423,0.4794255386$, $0.5646424733,0.6442176872,0.7173560908,0.7833269096$, $0.8414709848], \quad[1, \quad 0.9950041652, \quad 0.9800665778$, $0.9553364891,0.9210609940,0.8775825618,0.8253356149$, $0.7648421872,0.6967067093,0.6216099682,0.5403023058]\}$. The error $<0.005$.

\section{Conclusions}

The proposed method is a simple and effective procedure for solving nonlinear Volterra integral equations, as well as a 
system of nonlinear Volterra integral equations. The methods can be adapted easily to the Volterra integral equations of the first kind, which have the form $g(x)=\int_{A} K(x, t) y(t) d t$. The convergence rate could be higher if we use more complicated orthonormal or cardinal splines. Nevertheless, the resulting system of coefficients will be more complicated nonlinear systems, which could take more time and effort to solve.

\section{Conflict of Interests}

The authors declare that there is no conflict of interests regarding the publication of this paper.

\section{Acknowledgments}

The work was funded by the Natural Science Foundation of Anhui Province of China under Grant no. 1208085MA15 and the Major Project of the Nature Science Foundation of the Education Department, Anhui Province, under Grant no. KJ2014ZD30.

\section{References}

[1] I. J. Schoenberg, “On trigonometric spline interpolation," Journal of Mathematics and Mechanics, vol. 13, pp. 795-825, 1964.

[2] T. Lyche, L. L. Schumaker, and S. Stanley, "Quasi-interpolants based on trigonometric splines," Journal of Approximation Theory, vol. 95, no. 2, pp. 280-309, 1998.

[3] T. Lyche and R. Winther, "A stable recurrence relation for trigonometric B-splines," Journal of Approximation Theory, vol. 25, no. 3, pp. 266-279, 1979.

[4] A. Sharma and J. Tzimbalario, "A class of cardinal trigonometric splines," SIAM Journal on Mathematical Analysis, vol. 7, no. 6, pp. 809-819, 1976.

[5] X. Liu, "Bivariate cardinal spline functions for digital signal processing," in Trends in Approximation Theory, K. Kopotum, T. Lyche, and M. Neamtu, Eds., pp. 261-271, Vanderbilt University, Nashville, Tenn, USA, 2001.

[6] X. Liu, "Univariate and bivariate orthonormal splines and cardinal splines on compact supports," Journal of Computational and Applied Mathematics, vol. 195, no. 1-2, pp. 93-105, 2006.

[7] X. Liu, "Interpolation by cardinal trigonometric splines," International Journal of Pure and Applied Mathematics, vol. 40, no. 1, pp. 115-122, 2007.

[8] R. Kress, Linear Integral Equations, vol. 82 of Applied Mathematical Sciences, Springer, Berlin, Germany, 1989.

[9] B. L. Moiseiwitsch, Integral Equations, Dover, New York, NY, USA, 2005.

[10] A. D. Polyanin, Handbook of Integral Equations, CRC Press, Boca Raton, Fla, USA, 1998.

[11] A. Vahidian Kamyad, M. Mehrabinezhad, and J. Saberi-Nadjafi, "A numerical approach for solving linear and nonlinear volterra integral equations with controlled error," IAENG International Journal of Applied Mathematics, vol. 40, no. 2, pp. 69-75, 2010.

[12] J. Goncerzewicz, H. Marcinkowska, W. Okrasiński, and K. Tabisz, "On the percolation of water from a cylindrical reservoir into the surrounding soil," Zastosowania Matematyki, vol. 16, no. 2, pp. 249-261, 1978.
[13] D. Wei, "Uniqueness of solutions for a class of non-linear volterra integral equations without continuity," Applied Mathematics and Mechanics, vol. 18, no. 12, pp. 1191-1196, 1997. 


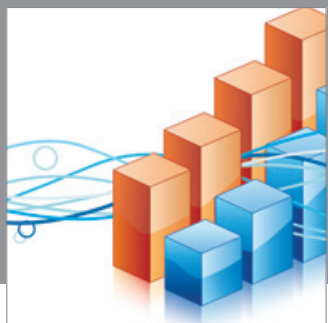

Advances in

Operations Research

mansans

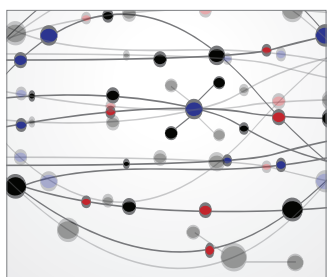

The Scientific World Journal
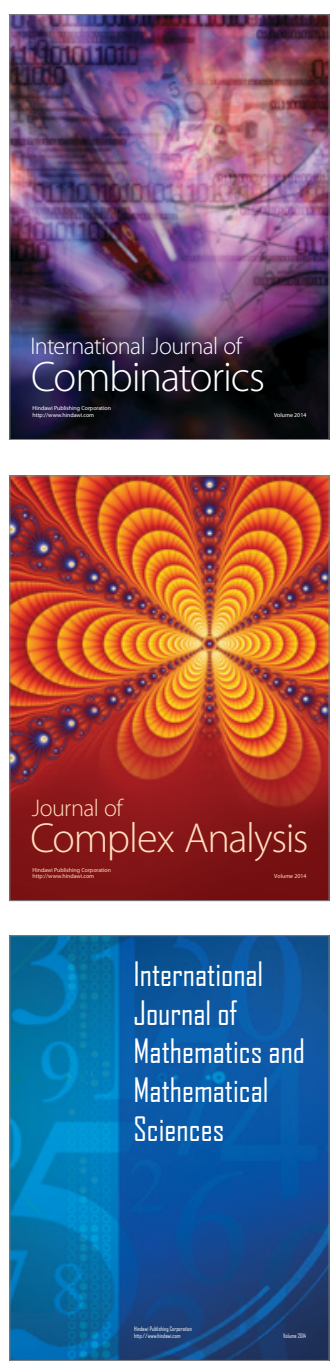
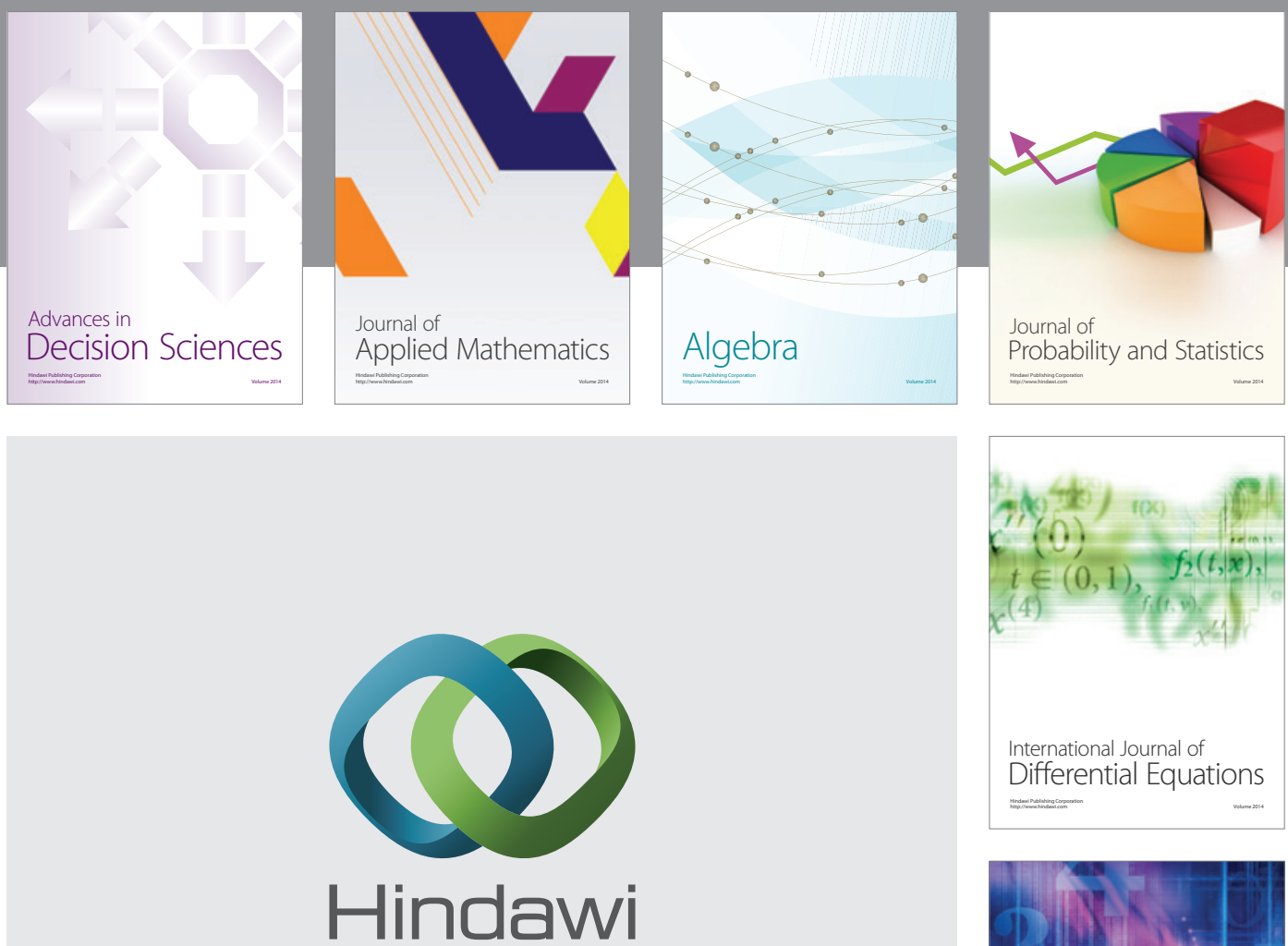

Submit your manuscripts at http://www.hindawi.com
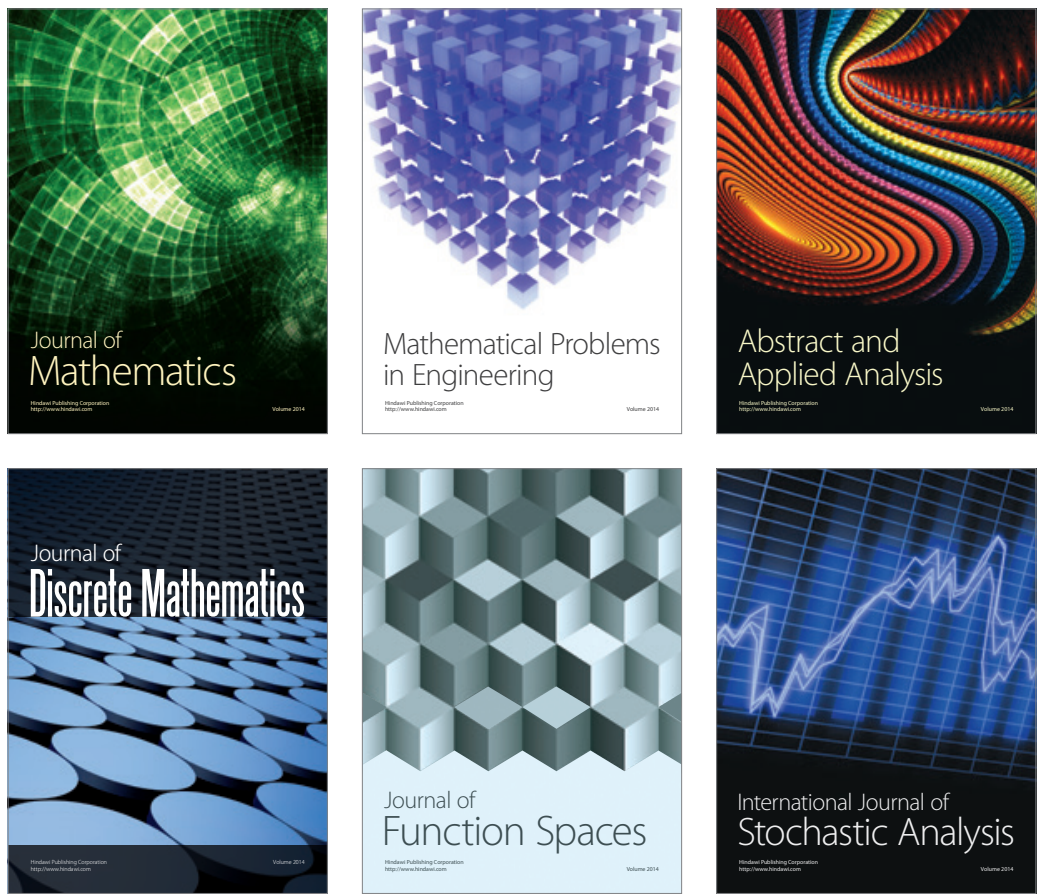

Journal of

Function Spaces

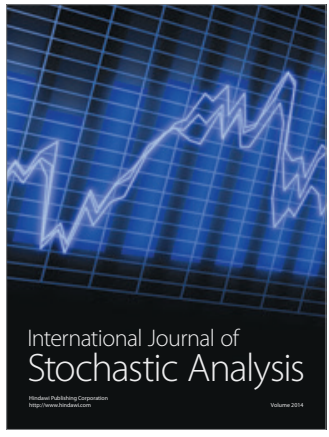

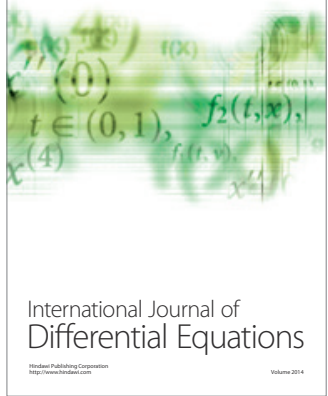
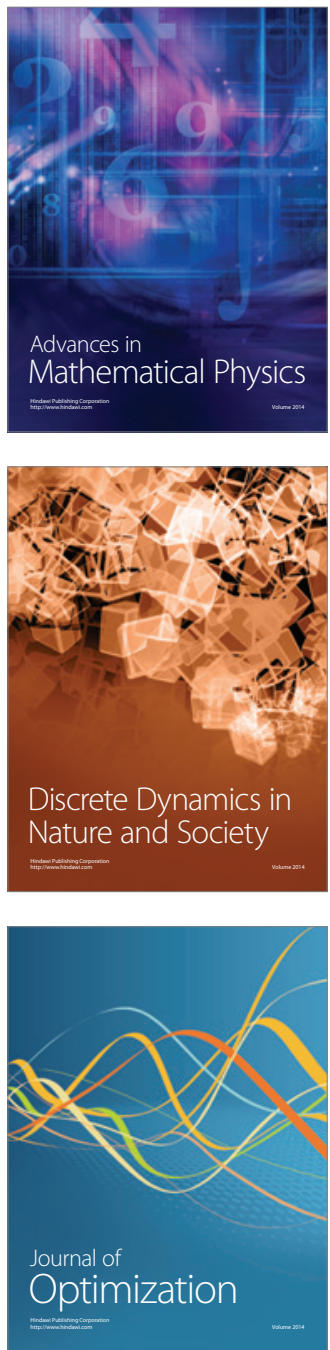